\title{
Analysis of the oscillation behavior during ultrasonic welding of EN AW-1070 wire strands and EN CW004A terminals
}

\author{
Andreas Gester $^{1}\left(\mathbb{D} \cdot\right.$ Guntram Wagner $^{1} \cdot$ Pascal Pöthig $^{2} \cdot$ Jean Pierre Bergmann ${ }^{2} \cdot$ Marco Fritzsche $^{3}$
}

Received: 6 September 2021 / Accepted: 22 November 2021 / Published online: 28 November 2021

(c) The Author(s) 2021

\begin{abstract}
For fulfilling the demand of durable yet lightweight electrical connections in transportation industries, ultrasonic metal welding (USMW) sees widespread use in these branches. As the ultrasound oscillations utilized in the welding procedure occur at a range of only a few micrometers at frequencies of $20-100 \mathrm{kHz}$ for an overall duration of only $50-1500 \mathrm{~ms}$, it is not possible to observe the compaction behavior with the bare eye. This paper focusses on investigating the oscillation behavior of the horn, the anvil, and the joining partners during the welding procedure by utilizing an array of synchronized laser vibrometers and performing welds with incrementing time stages. The oscillation data is correlated with temperature measurements in the welding zone as well as tensile testing results. Inter alia the formation of sidebands at the fundamental frequency as well as 2nd- and 3rd-order harmonics has been observed for the anvil, terminal, and wire front face when exceeding optimal weld time which would lead to maximum joint strength. Following the assumption of other research groups, the cause of these sidebands could be a change in relative motion of these components. As the terminal is slipping with increasing weld time, it could be assumed that the reason for the sidebands is low-frequency movement of the anvil, modulated onto the fundamental frequency, additionally indicating successful bonding of the stranded wire and the terminal. Furthermore, this slipping of the terminal on the anvil could lead to increased wear of the anvil knurls.
\end{abstract}

Keywords Ultrasonic metal welding $\cdot$ Aluminum $\cdot$ Copper $\cdot$ Dissimilar joints $\cdot$ Laser vibrometry $\cdot$ Sidebands

\section{Introduction}

Recommended for publication by Commission III - Resistance Welding, Solid State Welding, and Allied Joining Process

Andreas Gester

andreas.gester@mb.tu-chemnitz.de

Guntram Wagner

guntram.wagner@mb.tu-chemnitz.de

Pascal Pöthig

pascal.poethig@tu-ilmenau.de

Jean Pierre Bergmann

jeanpierre.bergmann@tu-ilmenau.de

Marco Fritzsche

m.fritzsche@polytec.de

1 Chair of Composites and Material Compounds, Chemnitz University of Technology, Chemnitz, Germany

2 Department of Production Technology, Ilmenau University of Technology, Ilmenau, Germany

3 Polytec GmbH, Waldbronn, Germany
Ultrasonic metal welding (USMW) is widely used in transportation industries, e.g., automotive, shipbuilding, aerospace, or railway construction. In these branches, USMW mostly sees application for producing electrically conductive joints like bus bar connections, wire harnesses, or battery leads [1]. Current trends in e-mobility and lightweight structures however pursue contradictory interests, on the one hand demanding weight reduction of these electrical connections in the vehicle, and on the other hand requiring increasing current carrying capacity due to improving battery and drive technologies. To encounter this, copper wires were substituted by aluminum in the last years. However, as the electrical conductivity of aluminum is considerably lower than that of copper, the aluminum wire has to exhibit a cross section amounting to $160 \%$ of that of the copper wire for achieving the same conductivity [2]. Due to the lower density of aluminum, this substitution still results in a weight reduction of approximately $40 \%$ [3]. Table 1 compares these properties of copper and aluminum. 
Table 1 Density and electrical conductivity of copper and aluminum [4]

\begin{tabular}{lll}
\hline Element & $\begin{array}{l}\text { Electrical conductivity } \\
\left(\mathrm{m} /\left(\Omega * \mathrm{~mm}^{2}\right)\right)\end{array}$ & $\begin{array}{l}\text { Density } \\
\left(\mathrm{g} / \mathrm{cm}^{3}\right)\end{array}$ \\
\hline Copper & 8.93 & 58 \\
Aluminum & 2.70 & 35.50 \\
\hline
\end{tabular}

This increase in wire diameter of such a dissimilar joint of aluminum wire and copper terminal poses a challenge for conventional joining techniques, e.g., soldering or fusion welding processes. Copper and aluminum are prone for the formation of a large variety of intermetallic compounds (IMC), which exhibit increased brittleness and decreased electrical conductivity, thus negatively influencing the mechanical as well as electrical properties of the joints [5]. Additional problems emerge for joining methods, where no fusion occurs at the interface, e.g., crimping, as copper and aluminum develop passivation layers on their respective surfaces while being exposed to oxygen [6]. These passivation layers reduce the contact area of the pure metals in the joining zone, which causes increased contact resistance further leading to decreasing current carrying capacity as high electrical currents result in significant heating of the joint in practice.

In solid-state joining technologies such as USMW, these aforementioned effects can be significantly mitigated or avoided completely. In USMW, the joining temperature lies below the melting temperature of the joining partners, approximately at $45 \%$ for similar joints of aluminum or copper and at $67 \%$ for dissimilar aluminum/copper joints [7]. In such a temperature range, the formation of IMCs at the interface of aluminum and copper cannot be avoided completely, but greatly reduced. Furthermore, at the start of the welding procedure, the ultrasonic excitation of the upper joining partner and the resulting friction between this part and the lower (clamped) joining partner are causing a dispersion of the oxide layers at the surface of the joining partners, enabling pure metal contact [8]. Other researchers, e.g., Zhang et al. [9, 10], have shown that not all of the passivation layer's oxides will be removed by the cleaning effect of the ultrasonic oscillations as they observed both residual oxides as well as segregations accumulating at the interface of both dissimilar materials which may act as diffusion barrier, preventing the growth of intermetallic compounds which, in the case of aluminum and copper as joining partners, would negatively impact interface conductivity and joint strength. A joint produced by USMW therefore exhibits superior conductivity at the interface in comparison to conventional joining methods [11].

USMW can be used for various geometries, ranging from foils and sheets to wires, whereas welding of the latter poses a complex task as the wire consists of multiple strands with amounts ranging from one up to four digits depending on the wire configuration and the cross section. Thus, the behavior of the wire resembles that of a highly elastic and flexible joining partner with additional degrees of freedom in contrast to rigid sheet metals.

Although USMW is widely used for this type of joints whereas ultrasonic oscillation is the main driver for the frictional heat, disruption of passivation layers, and other effects, only few literature exist on the investigation of the oscillation behavior of the USMW components during the joining of wires and terminals. Mostafavi et al. investigated the influence of terminal modifications on the time and frequency domains during the welding procedure of brass terminal/aluminum wire joints via piezoelectric transducers mounted on the terminals [12]. The signals observed also exhibited the existence of strong harmonics at multiples of 2 to 5 based on the fundamental frequency. Taking this into account, they recommended to simulate the eigenfrequencies of terminals not only in the band of the fundamental frequencies but also for the higher order harmonics in order to avoid damaging the joint or terminal itself. Furthermore, the research group observed the formation of sidebands at the fundamental frequency and higher order harmonics for the first time. They assumed that the formation of sidebands during USMW could indicate a failure in the weld, however mentioning that there exist only little knowledge of sidebands on the field of ultrasonic welding.

The research group of Abi Raad et al. investigated the US welding process of copper to copper sheets while measuring horn and anvil oscillations with out-of-plane laser vibrometers as well as airborne sound with a 0.25 -in. microphone [13]. The researchers also observed the formation of higher order harmonics at the measurement positions on the anvil and the horn as well as for the acoustic emissions received by the microphone. Furthermore, they filtered the vibrometers' time domain signals by the main frequency bands and segmented these signals into discrete welding stages and could correlate expected behavior of horn, anvil, and joining partners with the measurements. However, acoustic emissions did not correlate with the vibrometer signals.

A research group of Balz et al. also utilized laser vibrometers with measurement locations on the horn and the anvil as well as several other sensor sources, e.g., machine data, high-speed imaging, and temperature measurements in order to better understand the behavior of the horn and anvil during USMW of copper sheets [14]. They found that the higher order harmonics are sensitive to influences in the welding process. Additionally, it has been observed that measuring the oscillations of the anvil was less prone to error and could possibly help in feature detection when used for process monitoring. 
As literature review has not revealed any investigation on simultaneous oscillations measurements of all components during USMW - especially not for joints of stranded wires and terminals - this work addresses a better understanding of the oscillation behavior of such joints. Additionally, as Balz et al. have shown, the USMW process is highly sensitive to influences during the welding taking place [14]. In order to not influence the complex system during data acquisition, a non-tactile measurement would be the preferred choice. Therefore, multiple in-plane as well as out-of-plane vibrometers have been utilized to record the parts' oscillations during USMW.

\section{Experimental procedure}

In these investigations, terminals of EN CW004A with dimensions of $50 \times 22.5 \times 3 \mathrm{~mm}(\mathrm{~L} \times \mathrm{W} \times \mathrm{H})$ have been used as the lower joining partner. As the upper joining partner, PVC-insulated stranded wires of EN AW-1070 with a cross section of $50 \mathrm{~mm}^{2}$ have been used. Before welding, the terminals were degreased with ethanol, etched with a solution $10 \%$ sulfuric acid and water, and subsequently rinsed in deionized water. For performing the welds, a USMW system Telsonic MT8000 with a generator power of $10 \mathrm{~kW}$ has been used, which utilizes the PowerWheel principle in which the horn is excited torsionally, resulting in a horn movement in a circular segment. Figure 1 shows the structure of the welding

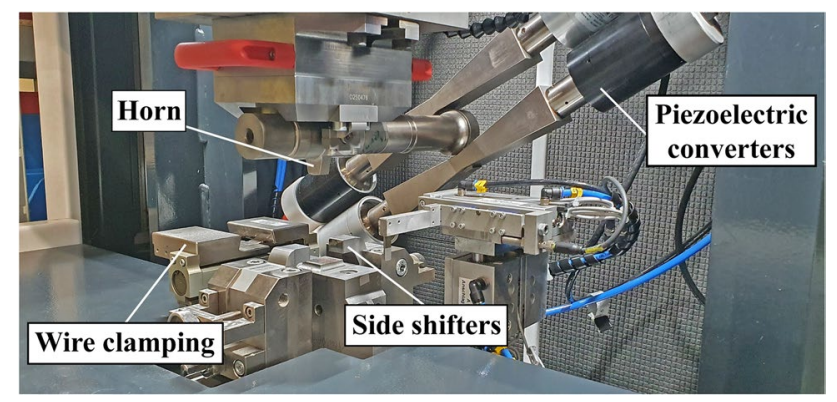

Fig. 1 Structure of the welding system system. The terminal was clamped via side shifters whereas the wire was fixed with a separate clamping.

The welding parameters used for these experiments were identified via a face-centered central composite experimental design with the response being the shear strength of the joint. In the experiments of this investigation, the weld time was increased incrementally by steps of $100 \mathrm{~ms}$, starting at $0.2 \mathrm{~s}$, and ending at $1.5 \mathrm{~s}$. Each welding procedure was repeated 3 times. For measuring the temperature in the weld zone, sacrificial type $\mathrm{K}$ thermocouples were inserted in the interface between the wire and the terminal. A 3D printed mask was used for positioning the terminal as well as the thermocouples to ensure a high level of reproducibility. Figure 2 a shows an isometric view of the mask for positioning both elements and aligning them to the position of the side shifters. Kepton tape has been used for fixing the location of the thermocouple, once positioned. Figure $2 \mathrm{~b}$ shows a side view of the thermocouple position. The joined connection of both thermocouple strands was positioned in such a way that the center of the horn was located directly above the thermocouple.

The laser vibrometers used in these investigations were one in-plane-vibrometer Polytec IPV-100, three out-ofplane-vibrometers Polytec Vibroflex, and one additional out-of-plane-vibrometer PSV-500. Figure 3a shows the arrangement of the vibrometers. Figure $3 \mathrm{~b}$ depicts their corresponding measurement points which were not altered during the experiments. All vibrometers were synchronized via a mutual controller with a sampling rate of $250 \mathrm{kHz}$ per channel. A high-pass filter with a cutoff frequency of $1 \mathrm{kHz}$ was applied during data processing in order to reject lowfrequency noise caused by $\mathrm{AC}$ hum and machines in the vicinity of the USMW system.

Measurement point (MP) 1 was located on the horn in plunged state, MP 2 on the terminal, MP 3 on the anvil, MP 4 on the abutting face of the wire strands, and MP 5 on the shell surface of the wire. A section of $8 \mathrm{~mm} \times 5 \mathrm{~mm}$ has been cut out of the insulation for MP 5 and retroreflective tape was placed on the now visible strands in order to increase the signal to noise ratio (SNR) of vibrometer 5 . This section was placed at a distance of $50 \mathrm{~mm}$ from the front surface of the wire as the side shifters and the horn would obscure sight
Fig. 2 a Isometric view of the mask used for positioning the terminal and the thermocouple and $\mathbf{b}$ side view visualizing the placement of the thermocouple
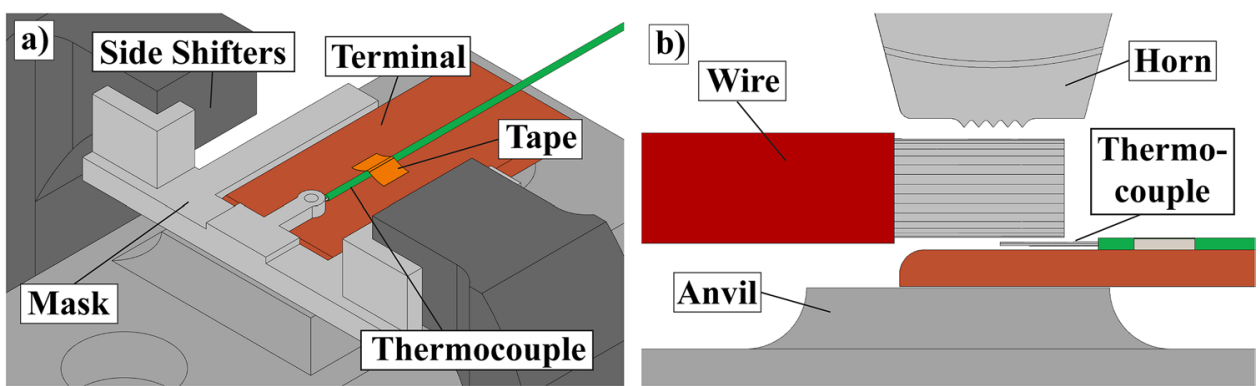
Fig. 3 a Vibrometers used (1, 2, 3: Vibroflex; 4: PSV-500; 5: IPV-100) and b corresponding measurement points (1: horn; 2 : terminal; 3 : anvil; 4 : wire front surface; 5: wire shell surface)
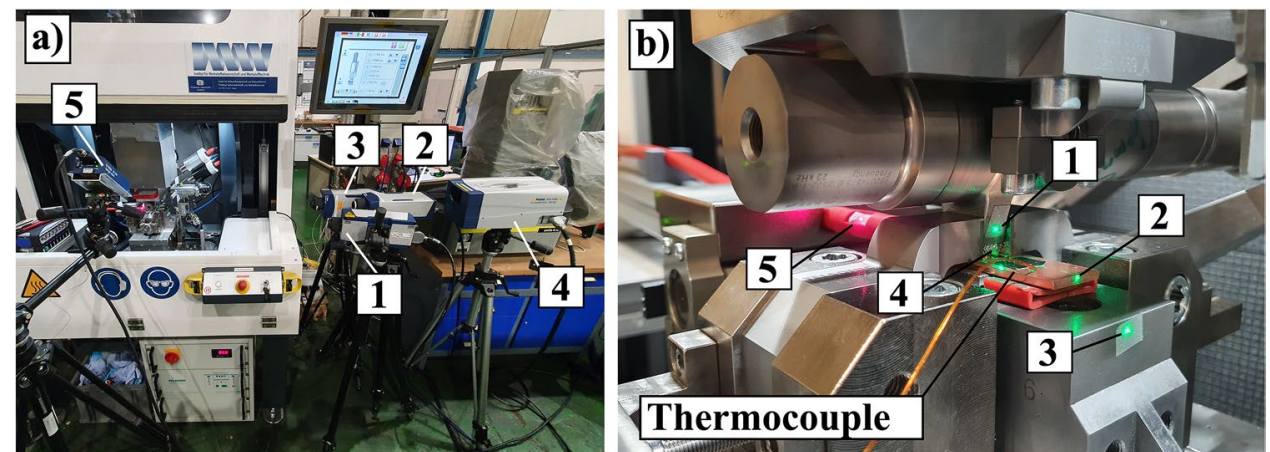

on the measurement location for shorter distances. The thermocouple signal has been recorded with an HBK Quantum MX809B thermocouple amplifier at a sample rate of $100 \mathrm{~Hz}$ with a $20-\mathrm{Hz}$ Bessel low pass filter applied. The amplifier was synchronized with the vibrometer controller. The shear strength of the joints has been determined with a Zwick/ Roell ProLine $10 \mathrm{kN}$ universal testing machine at a testing speed of $50 \mathrm{~mm} / \mathrm{min}$.

\section{Results and discussion}

In order to associate joint strength and weld temperature with the time sequence, a set weld time of $1500 \mathrm{~ms}$ was separated into $100-\mathrm{ms}$ increments. Therefore, the preliminarily identified optimal parameter set, with a weld time of $1000 \mathrm{~ms}$, is contained as well as under-welds (joints welded with insufficient frictional heat, resulting in poor joint strength) and over-welds (joints welded with excessive frictional heat, resulting in strong plastic deformation and also poor joint strength). Figure 4 shows the relation of weld time, joint shear strength, weld temperature, and ultrasound energy required as well as horn penetration for the respective time stage.
This joint configuration shows an increase of shear strength from 200 up to $1000 \mathrm{~ms}$ with the maximum joint strength at $1000 \mathrm{~ms}$. Subsequent time increments result in a decrease of joint strength, therefore classifying the welds with a weld time $\leq 900 \mathrm{~ms}$ into under-welds, at $1000 \mathrm{~ms}$ into optimal welds, and $\geq 1100 \mathrm{~ms}$ into over-welds. Temperature as well as ultrasound energy and horn penetration show a constant rise over the investigated time period. However, a drop in temperature and ultrasound energy is clearly visible at the time stage of $1100 \mathrm{~ms}$. Also, the horn penetration is forming a plateau in the time span of 1100 to $1200 \mathrm{~ms}$. This is in accordance to Balz et al. [14] and Abi Raad et al. [15], who observed slipping of the upper joining partner during the end of their welding process, whereas Mostafavi et al. [13] observed slipping between the lower joining partner and the anvil. It is assumed that this slipping is a result of the excessive heat input when exceeding optimal weld time, which causes increased plastic deformation and thinning of the aluminum. The temperature drop observed at $1100 \mathrm{~ms}$ is in accordance with Zhang [16], who assumed that this is a result of the reduced frictional heat. Balz et al. [14] supposed that the ultrasound excitation of the upper joining partner is reduced due its reduced strength, thus leading to a reduction in temperature. Additionally, Thomä and other
Fig. 4 Plot of joint shear strength, weld temperature, ultrasound energy, and horn penetration vs. weld time

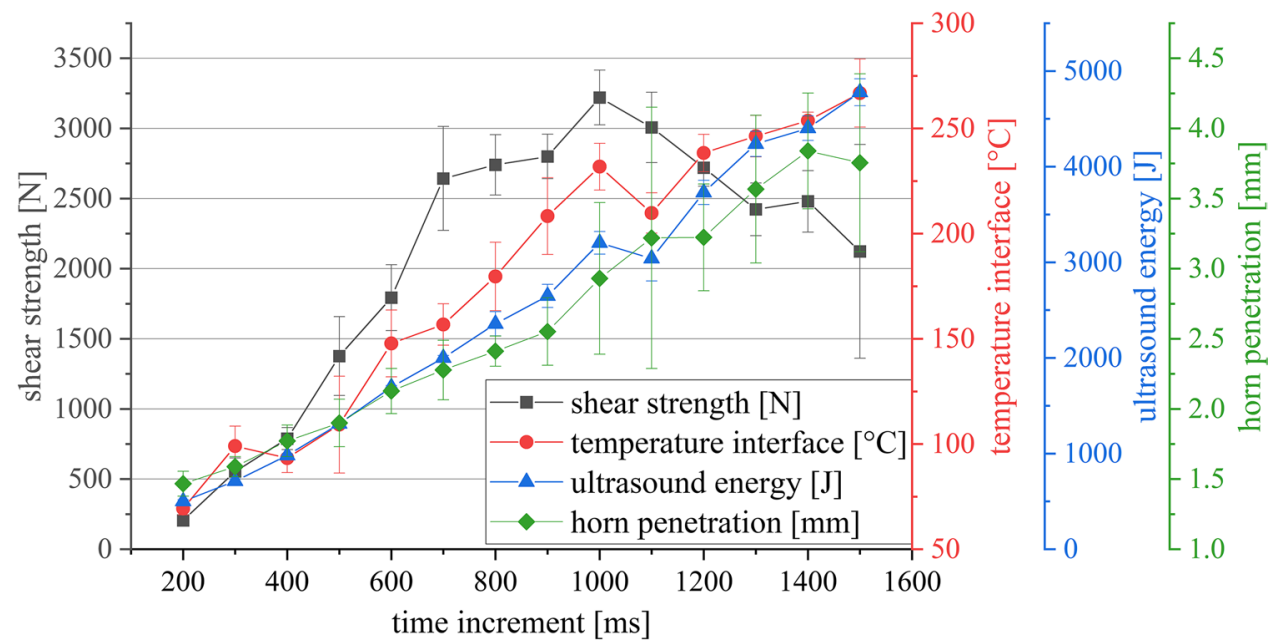


researchers showed the temperature dependence of the yield strength of aluminum (in this case AA6061) which exhibited a reduction of $72 \%$ at the recorded temperature range for the USMW process [17-19] which reinforces the assumption of Balz et al. [14]. For the time stages from 1200 to $1500 \mathrm{~ms}$, temperature and horn penetration depth increase again, while the joint strength drops further. It can be assumed that the aforementioned temperature drop results in slight increase in yield strength for the aluminum, leading to a better ultrasound excitation of the aluminum wire again. Furthermore, the recrystallization temperature of pure aluminum starts at $245^{\circ} \mathrm{C}$, which could lead to grain coarsening if the deformation of the aluminum is not adequate for promoting dynamic recrystallization at these time stages [20].

In the next step of the investigations, the oscillation measurements were performed. Figure 5 depicts the oscillation amplitudes of the welding system and the joining partners for an overwelded state with a weld time of $1.4 \mathrm{~s}$. For these plots, $20-\mathrm{kHz}, 40-\mathrm{kHz}$, and $60-\mathrm{kHz}$ bands were defined and filtered with a bandpass filter with cutoff frequencies amounting to $-1000 \mathrm{kHz}$ and $+1000 \mathrm{kHz}$ in respect to the aforementioned band frequencies. Whereas 40 and $60 \mathrm{kHz}$ amount to the respective higher order harmonics, $20 \mathrm{kHz}$ amounts to the fundamental frequency of the ultrasonic horn. As the amplitudes differ greatly from each other, no uniform scale of the amplitude axes was possible.

The horn oscillation, shown in Fig. 5a, starts with a sharp rise up to an amplitude of $22.5 \mu \mathrm{m}$ at $0.05 \mathrm{~s}$, which gradually decreases to $16 \mu \mathrm{m}$. However, as the welding system utilizes a torsionally driven horn, the measured amplitude decreases if the distance between the (stationary) measurement point and the center of the horn is reduced. Therefore, a constant
Fig. 5 Oscillation amplitudes of the welding system components and the joining partners
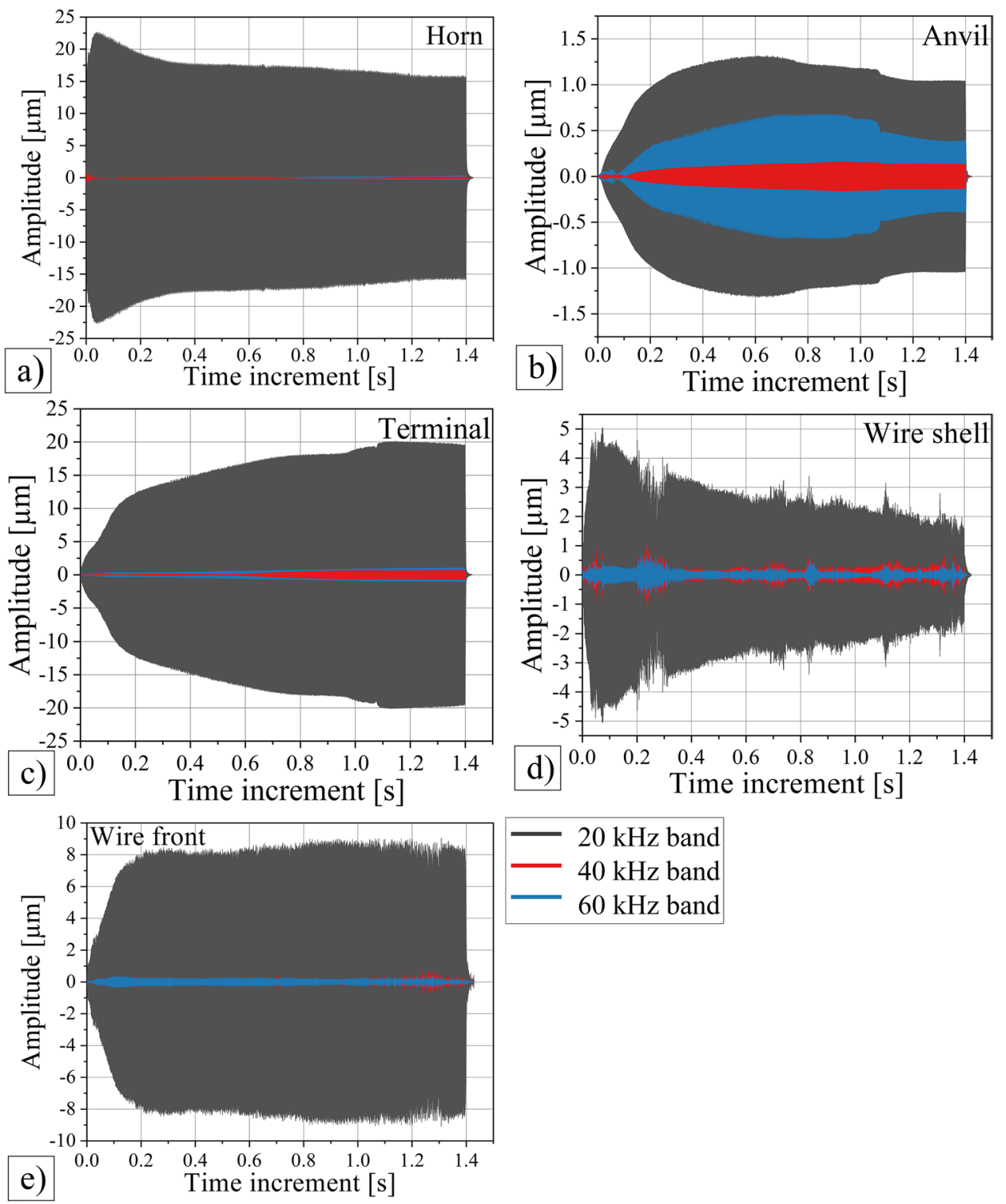

$20 \mathrm{kHz}$ band

$40 \mathrm{kHz}$ band

$60 \mathrm{kHz}$ band 
amplitude of the horn throughout the welding process can be assumed, which would be in accordance to Abi Raad et al. [13] and Balz et al. [14]. Figure 6 aims to visualize this relation.

The anvil oscillation, shown in Fig. 5b, exhibits a slower, parabolic rise up to its maximum amplitude of $1.25 \mu \mathrm{m}$ at $20 \mathrm{kHz}$ within $0.6 \mathrm{~s}$. The 2nd- and 3rd-order harmonics start to rise noticeably later at $0.1 \mathrm{~s}$, which is in contrast to [13], where the harmonics of the oscillations at the anvil started to rise nearly instantaneously with the ultrasound frequency of $20 \mathrm{kHz}$. The reason for this behavior needs further investigation, but it could be assumed that the reason of the low harmonic's amplitudes at weld times below $0.1 \mathrm{~s}$ lies in the coupling of the horn and the aluminum wire at the start of the process, which initially causes pure slipping of the aluminum wire on the terminal. As there are no non-linearities involved in this time stage, harmonics could be decreased. This would also explain the nearly non-existing harmonics of the terminal, seen in Fig. $5 \mathrm{c}$. Starting at $0.7 \mathrm{~s}$, the anvil oscillations decrease with a sudden drop at 1.1-s weld time. The overall decrease of the anvil oscillation could be explained by slipping of the terminal on the anvil as soon as bond formation occurs. In addition, the rather strong harmonics have to be noted, with the 3rd-order harmonics amplitude amounting to nearly $50 \%$ of that of the fundamental frequency at $20 \mathrm{kHz}$. A large amount of harmonics was also observed by [13]; however, at the 2nd-order harmonics at $40 \mathrm{kHz}$. The distinctive drop in amplitude is also visible at the $3 \mathrm{rd}$ but not the 2nd-order harmonics. The sudden drop can not be explained in time domain and requires further analysis in frequency domain. The terminal oscillations are depicted in Fig. 5c. The shape at the fundamental frequency exhibits a similar shape to that of the anvil's fundamental frequency up to 0.7 -s weld time. However, instead of the decrease in amplitude at $20 \mathrm{kHz}$, observed at the anvil, the terminal's amplitude increases further, reaching almost the amplitude of the horn. The discontinuity at 1.1-s weld time seen at the anvil also is visible at the terminal in form of a jump to a higher amplitude instead of a drop in amplitude. The continuous rise of the terminal's oscillation amplitude

a)

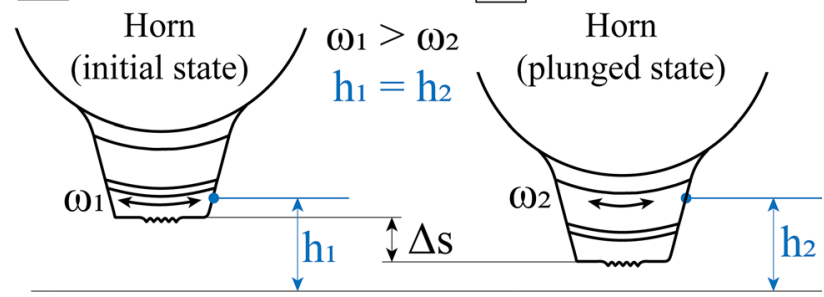

Fig. 6 Stationary measurement point position on the horn in a initial state and $\mathbf{b}$ plunged state can be explained by the increasing bond formation at the interface of the aluminum wire and the copper terminal. Thus, with progressing weld time, the terminal is increasingly excited by the horn's oscillation through the horn/wire coupling and increasing coupling of the wire and the terminal through bond formation. Analogous to the anvil, the sudden jump of the oscillation amplitude can not be explained in time domain. Figure 5 further shows the oscillations of the wire, measured in-plane on the wire shell (5d) and on the wire front face (5e). A non-uniform shape is observed for both waveforms, exhibiting large spikes. These could indicate a poor signal to noise ratio (SNR) or signal interruptions during measurement. As the strands in the aluminum wire are highly flexible, a large movement occurs at both measurement positions due to increasing consolidation of the wire in the welding process, complicating the measurement. Furthermore, the ejection of microscopic particle clouds during the welding procedure has been observed in earlier investigations utilizing high-speed imaging. It is assumed that these particle clouds consist of aluminumoxide and contaminants, whereas further investigations have to prove this. These particle clouds are ejected on the wire front surface where measurement point 4 was located, obfuscating the laser beam of the vibrometer, thus leading to distortion of the vibrometer signal. Interestingly, the oscillations at the wire shell exhibit a decrease in oscillation amplitude throughout the welding process whereas the oscillations on the front surface remain on a stationary level. It has been assumed by [14] that the decrease of amplitude over time for the upper joining partner is the result of the increasing plastification, leading to improper transfer of the ultrasonic oscillations at higher weld times. However, this is in contrast to the measured amplitudes on the wire front surface. The constant amplitude indicates sufficient coupling of the horn and the wire throughout the process. Following this indication, the reason for the falling amplitude at the wire shell is unknown and needs further research, but a possible explanation is the increasing attenuation of the oscillation at this measurement location due to increasing consolidation. The generally lower amplitudes in comparison to the horn amplitudes measured on the wire shell could also be explained by this attenuation. The reason for the lower amplitudes on the wire front is unknown; however, it could be assumed that the poor SNR is one cause for these observations.

Figure 7 shows the frequency domain of the recorded oscillations via fast Fourier transformation (FFT). For the FFT, a sampling rate of $1 \mathrm{E}-6 \mathrm{~S} / \mathrm{s}$ and a von-Hann window were used. Note the different scale on the amplitude axis.

Figure 7a shows the Fourier analysis of the horn oscillation. Clearly visible is the largest peak at the horn resonance frequency of $20 \mathrm{kHz}$ with hardly visible harmonics. Figure $7 \mathrm{~b}$ depicts the Fourier analysis of the anvil. The large 3rd-order harmonics observed in time domain are also 
Fig. 7 FFTs of the recorded time signals of the welding system components and the joining partners
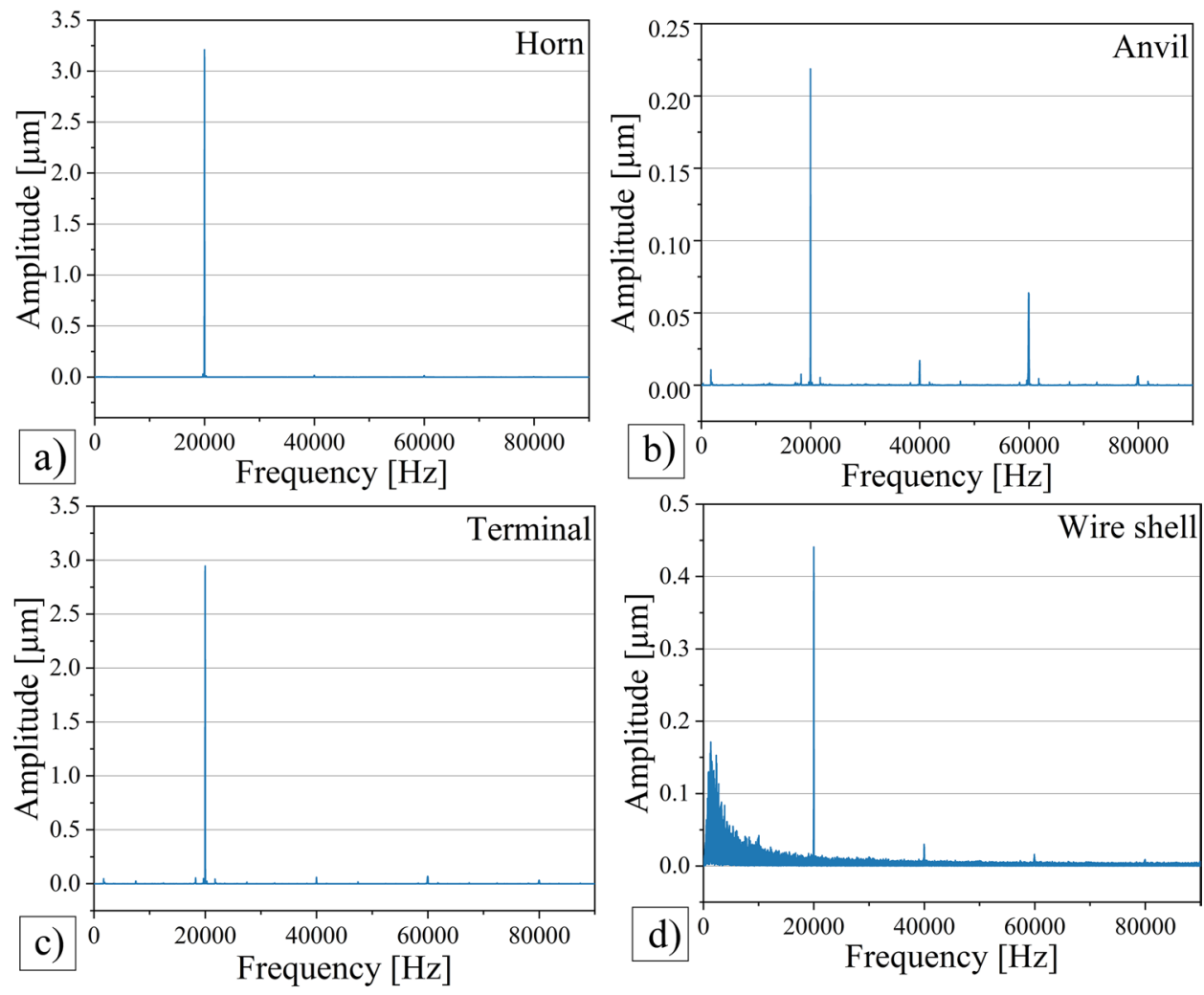

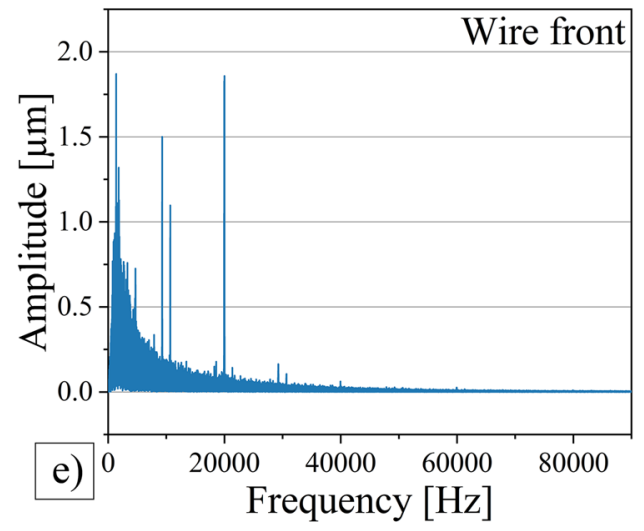

visible. Interestingly, the fundamental frequency and the harmonics exhibit the formation of sidebands at a distance of $+/-2 \mathrm{kHz}$ from the base frequency. These sidebands can also be observed for the terminal in Fig. 7c. The frequency analyses of the wire shell in Fig. 7d and the front surface in Fig. 7e exhibit similar noise floors, which was explained before by the particle clouds and large wire movement due to ultrasonic consolidation. The peaks at approximately $10 \mathrm{kHz}$ could be the result of single strands moving through the measurement point during consolidation. Additionally, for the wire front in Fig. 7e, sidebands can be observed above the noise floor.

In order to investigate the formation of the sidebands further, especially their temporal placement in the welding process, short-time Fourier transformation (STFT) was performed using a von-Hann window, a FFT length of 2048 samples, and a window length of 1024 samples. These settings provided a good compromise of time and frequency resolution in respect to Küpfmüller's uncertainty principle [21]. Figure 8 shows the STFT, performed for all aforementioned signals. Frequency axes are scaled to a section ranging from 17 to $23 \mathrm{kHz}$. Note the differing scales of the amplitude axes.

Figure 8a depicts the STFT results of the horn oscillation. The shape of the waveform at $20 \mathrm{kHz}$ resembles that of the time domain signal in Fig. 5a. This is expected as the Fourier analysis shown in Fig. 7a indicated that the total vibrations of the horn comprise mostly the fundamental frequency at $20 \mathrm{kHz}$. The STFT results of the anvil as well as the terminal shown in Fig. $8 \mathrm{~b}$ and $\mathrm{c}$ exhibit the sidebands observed in the 
Fig. 8 STFT results of the recorded time signals of the welding system components and the joining partners
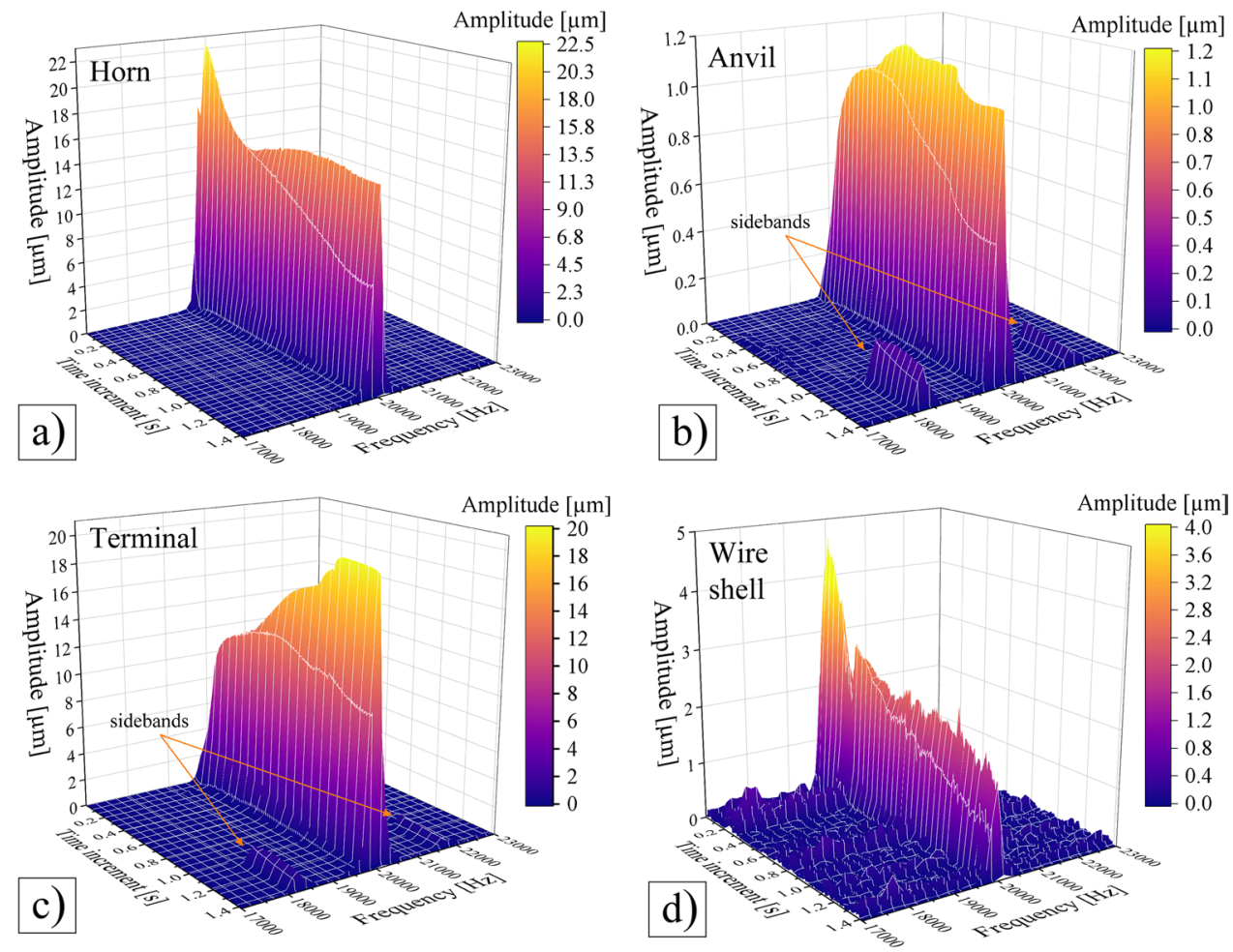

Amplitude $[\mu \mathrm{m}]$

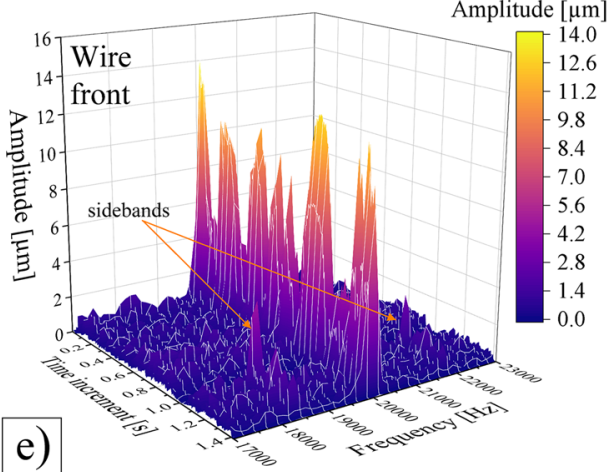

Fourier analysis, depicted in Fig. 7, which are located symmetrical around the base frequency at 18.3 and $21.7 \mathrm{kHz}$. These sidebands emerge at a weld time of $1.1 \mathrm{~s}$ and correlate with the drop or jump of amplitude observed at the time domain signals of anvil and terminal. The wire shell STFT result in Fig. 8d exhibits a strong noise floor around the fundamental frequency of the ultrasound. Apart from that, no sidebands or other anomalies could be found for this spectrum, even though the possibility exists that the sidebands are superimposed by the noise floor. In the STFT results of the wire front (Fig. 8e), the existence of sidebands is observed again, however strongly distorted due to a weak signal to noise ratio.

According to literature review, only Mostafavi et al. [12] observed sidebands in the oscillations during the welding process; however, it has to be noted that only few research groups investigated the oscillation behavior of one or more components during USMW of terminals and stranded wires. As Balz et al. [14] as well as Abi Raad et al. [13] did not observe sidebands, the assumption can be made that the emergence of sidebands depends on the geometry and/or the material of the joining partners. Sidebands are well-known characteristics in electromagnetic waves, e.g., amplitude or frequency modulation in radio technology. Mostafavi draws his conclusions from an investigation on sidebands forming in the oscillations of planetary gear sets by Inalpolat and Kahraman [22]. Inter alia Mostafavi translated the findings of this work into the observations he made at his investigations. He proposed that sidebands are occurring during USMW when a change of relative motion between components is taking place [12]. Following this assumption, the relative motion between anvil, terminal, and wire changes when increasing the weld time beyond the optimal parameter set, thus performing over-welding, as sidebands 
were detected for these components. A possible explanation could be the fact that the terminal is still clamped onto the anvil, even though micro-bonds are forming at the interface of the wire and the terminal. Thus, the terminal is increasingly excited by the oscillations of the horn. As time domain signals of the anvil and the terminal have shown, the anvil is effectively oscillating with the horn's amplitude with increasing weld time whereas the anvil exhibits only small oscillations. Thus, slipping of the terminal on the anvil has to occur inevitably, which could potentially lead to excessive wear of the anvil. Low-frequency movement of the anvil in this slipping stage could modulate onto the fundamental frequency, causing the observed sidebands. As slipping of the terminal could be assumed as successful bonding between wire and terminal, their appearance could be utilized as an indicator for process monitoring, which is also the conclusion of Mostafavi [12].

\section{Conclusions}

Joints of EN AW-1070 stranded wires with a cross section of $50 \mathrm{~mm}^{2}$ and EN CW004A terminals were produced via ultrasonic metal welding. The oscillations of the stranded wire, terminal, and anvil as well as the horn were measured via in-plane as well as out-of-plane laser vibrometry and analyzed in time and frequency domains as well as after short-time Fourier transformation. Welds were performed while incrementing the weld time from 0.2 up to $1.5 \mathrm{~s}$ in order to observe wire consolidation, ultrasonic energy, joint shear strength, and interface temperature as a function of weld time. The following conclusions could be drawn:

1. An optimum weld time of $1000 \mathrm{~ms}$ has been identified for this specific joint geometry. Exceeding this optimum, joint shear strength declines, possibly due to grain coarsening, excessive plastification, and thinning of the weld knot. Interface temperature drops slightly when exceeding the optimum weld time, whereas it is assumed that this temperature drop is the result of excessive plastification of the wire, leading to insufficient coupling of the horn and wire, thus resulting in reduced frictional heat between wire and terminal. When increasing the weld time further, temperature increases again, possibly due to now better coupling of the slightly cooled wire.

2. Analysis in time domain for the fundamental frequency band and 2nd as well as 3rd-order harmonics of the overwelded state showed that the terminal's amplitude approximates that of the horn with increasing weld time whereas the anvil's amplitude decreases and remains on a low level. The rising oscillation amplitude of the terminal can be explained by increasing bond formation at the interface between wire and terminal. Through this bond formation, the terminal starts to slip on the anvil, which explains the decrease in amplitude as well as the strong harmonics.

3. Both wire measurements exhibited poor SNR due to particle clouds ejected from the wire front and strong low-frequency movements of the wire during the welding process. The constant amplitude measured on the wire front is an indicator for a good coupling of the horn and the wire. On the wire shell, a falling amplitude was detected throughout the welding process. The exact reason for this behavior is unknown but could possibly be attributed to increasing attenuation of the oscillations with increasing consolidation at the 50-mm distanced measurement location.

4. Fourier analysis detected the emergence of sidebands in the vicinity of the fundamental frequency at $20 \mathrm{kHz}$ and the corresponding higher order harmonics. Furthermore, both wire measurements exhibit strong noise especially below $20 \mathrm{kHz}$, which could be attributed to the particle clouds and large wire movement, distorting the laser beams used for measuring.

5. Short-time Fourier transformation results showed that the sidebands observed in FFT start to emerge at a weld time of $1100 \mathrm{~ms}$, which is $100 \mathrm{~ms}$ beyond the optimum weld time. Possible explanations for the existence of these sidebands were formulated by Mostafavi et al. [12], e.g., that these sidebands indicate a change in relative motion between components, which would mean in the case of this investigations between wire, terminal, and anvil as only for these components were sidebands detected. It can be assumed that slipping of the terminal on the anvil is modulating low-frequency oscillations onto the fundamental frequency, thus indicating successful bonding between wire and terminal. The emergence of sidebands therefore could potentially be used for process monitoring or parameter search for unknown joint types.

Acknowledgements The investigations were supported by the "Arbeitsgemeinschaft industrieller Forschungsvereinigungen" AiF (AiF No. 11909/17). Thanks are due to Marco Fritzsche from Polytec GmbH for assisting with the measurements and providing the out-of-plane vibrometers.

Funding Open Access funding enabled and organized by Projekt DEAL.

\section{Declarations}

Conflict of interest The authors declare no competing interests.

Open Access This article is licensed under a Creative Commons Attribution 4.0 International License, which permits use, sharing, adaptation, distribution and reproduction in any medium or format, as long 
as you give appropriate credit to the original author(s) and the source, provide a link to the Creative Commons licence, and indicate if changes were made. The images or other third party material in this article are included in the article's Creative Commons licence, unless indicated otherwise in a credit line to the material. If material is not included in the article's Creative Commons licence and your intended use is not permitted by statutory regulation or exceeds the permitted use, you will need to obtain permission directly from the copyright holder. To view a copy of this licence, visit http://creativecommons.org/licenses/by/4.0/.

\section{References}

1. Matheny MP, Graff KF (2015) Ultrasonic welding of metals. In: Gallego-Juárez JA, Graff KF (eds) Power ultrasonics: applications of high-intensity ultrasound. Elsevier/WP Woodhead Publ, Amsterdam, pp 259-293

2. Bergmann JP, Petzoldt F, Schürer R et al (2013) Solid-state welding of aluminum to copper-case studies. Welding in the World 57(4):541-550. https://doi.org/10.1007/s40194-013-0049-z

3. Wagner G, Balle F, Eifler D (2010) Ultrasonic welding of aluminum wires with large cross sections. In: The Japan Institute of Light Metals (ed) Proceedings of the $12^{\text {th }}$ International Conference on Aluminium Alloys, September 5-9, 2010, Yokohama, Japan, pp 934-939

4. Braunovic M, Konchits VV, Myshkin NK (2007) Electrical contacts: fundamentals, applications and technology. Electrical and computer engineering, vol 132. CRC Press, Boca Raton

5. Kah P, Vimalraj C, Martikainen J et al. (2015) Factors influencing $\mathrm{Al}-\mathrm{Cu}$ weld properties by intermetallic compound formation. Int J Mech Mater Eng 10(1). https://doi.org/10.1186/ s40712-015-0037-8

6. Hoppe C, Ebbert C, Voigt M et al (2016) Molecular engineering of aluminum-copper interfaces for joining by plastic deformation. Adv Eng Mater 18(6):1066-1074. https://doi.org/10.1002/adem. 201500501

7. Balz I (2020) Prozessanalyse der thermo-mechanischen Vorgänge während der Verbindungsbildung beim Metall-Ultraschallschweißen, 1. Auflage. Aachener Berichte Fügetechnik, vol 2020,3. Shaker, Düren

8. Al-Sarraf Z, Lucas M (2012) A study of weld quality in ultrasonic spot welding of similar and dissimilar metals. J Phys: Conf Ser 382:12013. https://doi.org/10.1088/1742-6596/382/1/012013

9. Zhang CQ, Liu W (2019) Non-parabolic Al3Ti intermetallic layer growth on aluminum-titanium interface at low annealing temperatures. Mater Lett 256:126624. https://doi.org/10.1016/j. matlet.2019.126624

10. Zhang C, Robson JD, Haigh SJ et al (2019) Interfacial segregation of alloying elements during dissimilar ultrasonic welding of AA6111 aluminum and Ti6Al4V Titanium. Metall and Mater Trans A 50(11):5143-5152. https://doi.org/10.1007/ s11661-019-05395-7
11. Dhara S, Das A (2020) Impact of ultrasonic welding on multi-layered $\mathrm{Al}-\mathrm{Cu}$ joint for electric vehicle battery applications: a layerwise microstructural analysis. Mater Sci Eng, A 791:139795. https://doi.org/10.1016/j.msea.2020.139795

12. Mostafavi S, Hesser DF, Markert B (2019) Detection of terminal oscillation pattern in ultrasonic metal welding. J Manuf Process 41:159-167. https://doi.org/10.1016/j.jmapro.2019.03.035

13. Abi Raad E, Balz I, Reisgen U et al. (2019) Investigation of the applicability of acoustic emission and vibration analysis to describe the thermo-mechanical mechanism during ultrasonic metal welding. In: Ochmann M, Vorländer M, Fels J (eds) Proceedings of the $23^{\text {rd }}$ International Congress on Acoustics: Integrating $4^{\text {th }}$ EAA Euroregio 2019 : 9-13 September 2019 in Aachen, Germany. Deutsche Gesellschaft für Akustik, Berlin, Germany, pp 4700-4707

14. Balz I, Raad EA, Rosenthal E et al (2020) Process monitoring of ultrasonic metal welding of battery tabs using external sensor data. Journal of Advanced Joining Processes 1:100005. https:// doi.org/10.1016/j.jajp.2020.100005

15. Zhang G, Takahashi Y, Heng Z et al (2015) Ultrasonic weldability of $\mathrm{Al}$ ribbon to $\mathrm{Cu}$ sheet and the dissimilar joint formation mode. Mater Trans 56(11):1842-1851. https://doi.org/10.2320/mater trans.M2015251

16. Zhang $C$ (2011) A thermomechanical analysis of an ultrasonic bonding mechanism. Dissertation, Utah State University

17. Thomä M Wirkung von Leistungsultraschall auf das Prozessverhalten und die Bindungsmechanismen beim Rührreibschweißen von Aluminium/Stahl-Verbunden

18. ASM Handbook Committee (1990) Properties and selection: nonferrous alloys and special-purpose materials. ASM International

19. Wernicke S, Thier U, Hahn M et al (2020) Controlling material flow in incremental sheet-bulk metal forming by thermal grading. Procedia Manufacturing 50:257-264. https://doi.org/10.1016/j. promfg.2020.08.048

20. Vandermeer R, Juul Jensen D (2001) Microstructural path and temperature dependence of recrystallization in commercial aluminum. Acta Mater 49(11):2083-2094. https://doi.org/10.1016/ S1359-6454(01)00074-X

21. Schneider A, Mores R (2013) Fourier-Time-Transformation (FTT), Analysis of sound and auditory perception. In: Bader R (ed) Sound - perception - performance, vol 1. Springer International Publishing. Imprint; Springer, Heidelberg, pp 299-329

22. Inalpolat M, Kahraman A (2009) A theoretical and experimental investigation of modulation sidebands of planetary gear sets. J Sound Vib 323(3-5):677-696. https://doi.org/10.1016/j.jsv.2009. 01.004

Publisher's note Springer Nature remains neutral with regard to jurisdictional claims in published maps and institutional affiliations. 